Revue d'histoire de l'enfance « irrégulière »

Le Temps de l'histoire

9 | 2007

Violences et jeunesse

\title{
Jeunes et violences : pour une histoire de rapports
} de force...

Xavier Rousseaux

\section{(2) OpenEdition \\ 12 Journals}

Édition électronique

URL : http://journals.openedition.org/rhei/2173

DOI : 10.4000/rhei.2173

ISBN : 978-2-7535-1648-9

ISSN : 1777-540X

Éditeur

Presses universitaires de Rennes

Édition imprimée

Date de publication : 15 novembre 2007

Pagination : 127-140

ISSN : 1287-2431

Référence électronique

Xavier Rousseaux, " Jeunes et violences : pour une histoire de rapports de force... », Revue d'histoire de l'enfance « irrégulière » [En ligne], 9 | 2007, mis en ligne le 01 novembre 2009, consulté le 03 décembre 2020. URL : http://journals.openedition.org/rhei/2173; DOI : https://doi.org/10.4000/rhei.2173 


\section{Jeunes et violences : pour une histoire de rapports de force...}

À lire les comptes rendus de ces affaires dans la presse, à propos de la violence, immédiatement fleurissent des expressions stéréotypées : «du jamais vu ", "sans précédent " ou des jugements catégoriques : "la violence augmente", notre société est devenue plus violente (...qu'autrefois). Sans détours, une liaison quasi automatique est établie entre la montée de la violence et les comportements de la jeunesse. Presque naturellement se répandent des conceptions telles que "les jeunes d'aujourd'hui sont plus violents... ", sans que l'on sache très bien s'ils sont plus violents que les "vieux " ou s'ils sont plus violents que les adultes ne l'étaient à leur âge. Les médias raffolent des images de jeunes de plus en plus violents de plus en plus jeunes alimentant l'impression d'un dérèglement du rapport de forces (au sens premier) entre générations et de l'équilibre de la société.

Le regard de l'historien s'avère précieux parmi les spécialistes de sciences humaines et sociales avec lequel il partage un certain nombre de prémisses méthodologiques et critiques : prise au sérieux de la question médiatique, attention portée à la description (dense) des phénomènes, aux représentations par les discours en tout genre, dégagement face au jugement moral immédiat, remise de l'événement dans le contexte individuel, du groupe, de la société et de ses structures, effort de conceptualisation à partir du décryptage du sens donné par les acteurs et de la vulgate de l'«opinion ».

En l'occurrence, sa spécificité réside dans la mise à distance de l'événement violent, en le replaçant dans une séquence historique plus ou moins longue, mais également dans la contextualisation des permanences et des ruptures, des évolutions et des structures, dans ce que le sens commun et la nécessité d'utiliser des catégories stables nous font simplifier en « la violence " et « la jeunesse ». La liaison entre «violences » et « jeunesses » invite donc
Xavier

Rousseaux $^{(1)}$
(1) Chercheur qualifié au FNRS, professeur

à l'université catholique de Louvain. Respon-

sable du Centre d'histoire du droit et de la justice.

Ce texte est issu

des travaux d'un séminaire doctoral mené à l'univer-

sité catholique de

Louvain. Je remercie

David Niget pour

ses précieuses remarques

sur cette contribution.

(2) Stephen D. White, «Repenser la violence : de 2000 à 1000 ",

Médiévales, 37, 1999, p. 99-113.

(3) Pieter Spierenburg, "Introduction ", in Sophie Body-Gendrot, Pieter Spierenburg, [eds.], Cultures of Violence,

Xavier Rousseaux / p. 127 à 140 


$\begin{array}{ll}\text { Berlin, Springer (sous } & \text { Gauvard, « De grâce } \\ \text { presse). } & \begin{array}{l}\text { especial ». Crime, État et } \\ \text { société en France à la }\end{array} \\ \begin{array}{l}\text { (4) Yves Michaud, } \\ \text { "Violence ", in }\end{array} & \text { fin du Moyen Âge, Paris, } \\ \text { Dictionnaire de philoso- } & \text { Sorbonne, 1991; Nathalie } \\ \text { phie, Paris, Encyclopædia } & \text { Davis, Pour sauver sa } \\ \text { Universalis et Albin } & \text { vie. Les récits de pardon au } \\ \text { Michel, 2000, p. 1941- } & \text { XVlème siècle, Paris, } \\ \text { 1954 ; Xavier Rousseaux, } & \text { Seuil, 1988; Isabelle } \\ \text { "Violence ", in Diction- } & \text { Paresys, Aux marges du } \\ \text { naire encyclopédique } & \text { royaume. Violence, justice } \\ \text { de théorie et de sociologie } & \text { et société en Picardie } \\ \text { du droit, Paris, 2ème } & \text { sous François Ier, Paris, } \\ \text { édition, 1993, p. 641-647. } & \text { Publications de la } \\ & \text { Sorbonne, 1998. }\end{array}$

(5) Jacques Rossiaud,

«Prostitution, jeunesse et société dans les villes du Sud-Est au XVème siècle ", in Annales.

Economies, sociétés, civilisations, 1976, n 2, p. 289325 ; article repris dans La prostitution médiévale, Paris, Flammarion, 1988.

(6) Robert Muchembled, La violence au village, sociabilité et comportements populaires en Artois du XVème au XVIème siècle, Turnhout, Brepols, 1989 ; Claude
(7) Claude Gauvard, Altan Gokalp, « Les conduites du bruit et leur signification à la fin du Moyen Âge : le charivari ", Annales. Économies, sociétés, civilisations, 1974 , p. 693-704 ; Jacques Le Goff, Jean-Claude Schmitt, [dir.], Le charivari, actes de la table ronde organisée à Paris (1977) par le CNRS et l'EHESS, Paris, Mouton, 1981 ; Norbert Schindler, « Les gardiens du désordre ", in Giovani Levi, Jean- les historiens à mettre en perspective critique et à replacer dans un contexte plus global ces évidences primaires. Deux courants majeurs traversent la réflexion historique sur la violence. Certains chercheurs assimilent tout rapport de domination à la violence, ${ }^{(2)}$ tandis que d'autres restreignent l'usage du mot à l'exercice de contraintes physiques sur le corps ou l'honneur d'un individu. ${ }^{(3)}$

L'extrême polysémie du terme de violence me fait préférer une définition restreinte du comportement sur le plan analytique. ${ }^{(4)}$ Est un acte de violence celui qui est considéré comme tel par un discours émanant des protagonistes, d'observateurs ou d'évaluateurs, à condition qu'il s'agisse d'un acte d'agression visant la personne : insultes, coups, blessures.

Les textes proposés aux lecteurs s'inscrivent dans une problématique de longue durée. J'essaierai donc tout d'abord d'esquisser une fresque de la place des jeunes dans la violence, tout au moins depuis la fin du Moyen Âge, en partant d'abord de la question de la violence, plus explicite que celle de la jeunesse.

À partir du XVIème siècle, des sources nombreuses, civiles et administratives, mettent le doigt sur le phénomène. Partons du célèbre article sur la prostitution médiévale de Jacques Rossiaud : ${ }^{(5)}$ il y met au jour des formes de " viols collectifs ", de « tournantes " dans le langage d'aujourd'hui, présentées comme une licence permise aux jeunes célibataires. On y trouve déjà la matrice des menaces juvéniles telles que véhiculées par les adultes : les jeunes sont en bande, ils boivent et écument l'espace nocturne, les filles insouciantes ou frondeuses sont leur objet de prédation. Mais l'assignation par les adultes aux jeunes de formes spécifiques de violence ne traduit-elle pas une plainte de leur part face à des comportements qui leur paraissent mettre en danger l'ordre établi ? 
Ces comportements collectifs traversent les siècles. On les retrouve dans les lettres de rémission des XVème-XVIIème siècles, ${ }^{(6)}$ on les retrouve dans les charivaris depuis la fin du Moyen Âge, jusqu'aux sorties de guerres du XXème siècle, dont les jeunes sont les initiateurs. ${ }^{(7)}$

Au XIXème siècle, dans les communautés rurales, les attouchements sexuels, voire les relations sexuelles contraintes (viol), pour autant qu'ils fussent commis entre jeunes du village, étaient traités avec une large compréhension par une partie de la population mâle, considérant ces privautés comme un nécessaire rite d'intégration à la communauté. ${ }^{(8)}$

La nécessité d'endiguer par des moyens collectifs la violence paraît une des caractéristiques de la société occidentale. Dès le haut Moyen Âge, les systèmes coutumiers inscrivent les heurts entre individus dans le cadre des solidarités familiales. Le groupe de base, famille, clan, lignage, est également au cœur des processus de régulation de cette violence. Les systèmes de la vengeance constituent des rituels codifiés et encadrés de réponse à l'agression. ${ }^{(9)} \mathrm{Il}$ s'agit de permettre la rétribution sans enclencher le cycle sans fin de la vengeance. Dans bien des régions d'Occident, la famille est le lieu de ce rituel et les jeunes hommes y jouent un rôle important. Le chef de la guerre, seul habilité à mener la guerre privée contre un clan, est souvent l'héritier mâle du clan de la victime.

Certaines coutumes imposent au fils aîné d'une victime d'homicide de prendre en charge l'homicide de vengeance. En Hainaut, la coutume du forjurement ${ }^{(10)}$ consistait en une procédure de serment par laquelle les parents d'un agresseur ayant fui le pays reniaient celui-ci, tandis que le fuyard jurait de ne plus revenir dans le pays ; ce qui devait empêcher l'homicide de vengeance sans perte de la face. Les parents bénéficiaient ainsi de la protection " publique » du Prince et ne pouvaient être attaqués légitimement par la famille de la victime. Cette pratique bénéficia aux autorités, mais parfois à la demande des aînés de famille ne supportant pas le poids moral de leur responsabilité de "chef de la vengeance ».

Le développement des communautés urbaines entraîne une mutation des systèmes de régulation des conflits. Dans les villes qui, en Italie du Nord, dans la vallée du Rhin ou en Flandre, s'émancipent du pouvoir seigneurial aux XIème-XIIème siècles, la violence des rixes collectives entre étrangers et bourgeois demeure le problème central des autorités urbaines. Ces dernières s'efforcent de limiter les effets de cette violence sans la stigmatiser, par un sys-
Claude Schmitt, [dir.],

Histoire des jeunes en

Occident, t. 1, Paris, Seuil,

1996, p. 277-329; Marc

Jacobs, "Charivari in

Vlaamse dorpen

(XVIIIde-XXste eeuw).

Macht, cultuur en symbo-

lisch geweld " [Le chari-

vari dans les villages

flamands (XVIIIème-

XXème s.). Pouvoir, cultu-

re et violence symbo-

lique], in Les structures $d u$

pouvoir dans les commu-

nautés rurales en Belgique

et dans les pays limitrophes

(XIIème-XIXème siècle),

Bruxelles, Crédit communal, 1988, p. 589-614 ;

Benoît Majerus, « La

guerre commence.

Bruxelles en août 1914 et

en mai 1940 ", in Nicolas

Beaupré, Anne Dumenil,

Christian Ingrao, [dir.],

I9I4-I945. L'ère de la guerre.

Violence, mobilisations,

deuil, t. 1, Paris, Agnès

Viénot, 2004, p. 85-105;

Idem, « Bruxelles, 1918 :

révolution et sortie de

guerre ", in Philipe

Chassaigne, Jean-Michel 
Largeaud, [dir.], Villes en guerre, Paris, Armand Colin, 2004, p. 196-207.

(8) Geoffroy Le Clercq, «Violences sexuelles, scandale et ordre public. Le regard du législateur, de la justice et des autres acteurs sociaux au

XIXème siècle ", in Revue belge d'histoire contemporaine, 1999, 29, 1-2, p. 5-53.

(9) Raymond Verdier, Jean-Pierre Poly, [dir.], Vengeance, pouvoirs et idéologies dans quelques civilisations de l'Antiquité, Paris, Éditions Cujas, 1984 ; Société des historiens médiévistes de l'enseignement supérieur public, Le règlement des conflits au Moyen Âge, Paris, Publications de la Sorbonne, 2001.

(10) Voir, pour le Hainaut, Jean-Marc Bury, De la guerre privée à l'ordre du prince : le forjurement en Hainaut (I200-I534),
Louvain-la-Neuve, 20002001, université catholique de Louvain (UCL), mémoire de licence en histoire, inédit ; Ève-Marie Halba, « Le forjurement dans les épopées et les chroniques du Nord. Approche comparée d'une ancienne pratique judiciaire ", in Emmanuelle Poulain-Gautret, JeanPierre Martin, Jean-Pierre Arrignon et Stéphane Curveiller, [dir.], Le Nord de la France entre épopée et chronique, Arras, Artois Presses Université, 2005.

(11) Xavier Rousseaux, «Politiques judiciaires et résolution des conflits dans les villes de l'Occident à la fin du Moyen Âge. Quelques hypothèses de recherche ", in Pratiques sociales et politiques judiciaires dans les villes de l'Occident à la fin du Moyen Âge, Rome, École française de Rome, 2007 (sous presse). tème d'interdiction de port d'armes, et d'amendes en cas d'infraction aux bans municipaux, ${ }^{(11)}$ mais surtout d'auto-dénonciation, de trêves obligatoires entre agresseurs et agressés et de processus de négociation et de réconciliation. Les rebelles à ces dispositions, généralement librement consenties par les bourgeois, encourent des poursuites criminelles et des peines extrêmement lourdes.

Dans ce premier modèle de régulation urbaine, la jeunesse est rarement prise en compte de manière directe. Mais l'analyse des sources de la pratique révèle clairement que les protagonistes de ces «bastons » sont des hommes jeunes, comme en témoignent leurs surnoms. ${ }^{(12)}$

Les études menées sur les guerres de factions en Italie du Nord (pensons aux Capulet et aux Montaigu) et dans les villes hollandaises consacrent ce modèle de rivalité urbaine entre clans patriciens rivaux ou entre patriciens et gens des métiers. ${ }^{(13)}$ Les valets de l'entourage d'un puissant, les bataillons d'artisans d'un métier constituent autant de forces jeunes et viriles destinées à mener la rivalité urbaine. Dès le Moyen Âge également, le lien entre groupe de jeunes, usage de la force physique et rituels urbains est établi, en particulier les activités physiques telles que tournois ou compétitions sportives, à l'instar du célèbre Palio de Sienne, course de chevaux annuelle entre milices de quartiers, dont les jockeys, souvent mercenaires, symbolisent une jeunesse brutale, frivole et incontrôlable. Or, comme les corporations d'artisans, les milices urbaines enrôlent les jeunes garçons dès l'âge de la puberté, soit 14 à 15 ans. La course sert de substitut à la violence possible entre les milices rivales.

Les filles sont moins présentes dans les représentations, ${ }^{(14)}$ non que la violence féminine ne soit pas prise en compte par les autorités urbaines médiévales, mais la jeunesse n'y est pas dénoncée comme élément fon- 
damental. C'est la violence verbale qui est assignée aux femmes comme forme socialement dangereuse, donc objet d'une intervention publique.

Les autorités s'efforcent d'utiliser la force physique des jeunes urbains au profit de la ville. Faute de corps de police professionnels, c'est à eux qu'il incombe de défendre les bourgeois agressés et de poursuivre les malfaiteurs étrangers devant les autorités urbaines. Ce sont eux qui contribuent aux milices urbaines et sont chargés de la garde aux remparts durant tout l'Ancien Régime. Néanmoins, vers la fin du Moyen Âge, leur présence devient parfois menaçante.

Cette évolution se trouve confirmée dans le texte d'Aude Musin et Élise Mertens de Wilmars, qui observent que les sources urbaines comme les lettres de rémission accentuent plus tardivement l'utilisation de l'argument de jeunesse comme moyen de défense. Face à la souveraineté du prince, la jeunesse devient progressivement un argument de défense. Deux raisons me semblent diriger ce phénomène. La disqualification progressive du comportement violent comme mode d'action collectif et le passage progressif de la responsabilité collective à la responsabilité individuelle. ${ }^{(15)}$

Les laboratoires de ces évolutions sont sans conteste les sociétés urbaines aux confins du Moyen Âge et des Temps modernes. En raison d'une sorte d'effet aspirateur des travaux sur la genèse de l'État, focalisés sur les institutions monarchiques et les Parlements, ces débats sont assez peu développés en France, sinon par des médiévistes comme Claude Gauvard ou Nicole Gonthier. Dans les régions d'Europe fort urbanisées, la documentation permet de prendre la mesure de cette autonomie des phénomènes urbains. Dès le XVème siècle, une nouvelle « culture urbaine " promeut une disciplinarisation croissante des groupes sociaux (Sozial-Disciplienierung) et même une culture de la " pudeur » qui s'oppose à la culture " de l'honneur ». ${ }^{(16)}$ Alors que Norbert Élias attribuait ce processus de disciplinarisation à une descente des valeurs de la noblesse vers les bourgeoisies, puis les classes populaires urbaines, les historiens des villes du bas Moyen Âge en font plutôt le produit d'une nouvelle « culture civique » des élites bourgeoises. Ce réveil éthique se manifeste par la poursuite, par les autorités, des sexualités déviantes ou des déviances verbales, par un contrôle plus tatillon des espaces et des temps des loisirs (bordels, tavernes, fêtes et jeux), par l'encadrement et l'urbanisation des rituels de fêtes de mariage, par la méfiance croissante envers l'étranger, le vagabond, l'hétérodoxe ou la sorcière. ${ }^{(17)}$
(12) Catharina Lis,

Hugo Soly, [dir.], Tussen dader en slachtoffer.

Jongeren en criminaliteit in historisch perspectief $[$ Entre auteur et victime. Jeunes et criminalité en perspective historique], Bruxelles,

VUB Press, 2001.

(13) Voir Nicole

Gonthier, Cris de haine et rites d'unité. La violence dans les villes. XIIèmeXIVème siècles, Turnhout, Brepols, 1992, Edward Muir, Mad Blood Stirring : Vendetta and Factions in Friuli during the Renaissance, Baltimore, John Hopkins UP, 1993 ; David Nicholas, The van Arteveldes of Ghent : The Varietie of Vendetta and the Hero in History, Ithaca, Cornell UP, 1988.

(14) Didier Lett, « Le corps de la jeune fille. Regards de clercs sur l'adolescente aux XIIèmeXIVème siècles ", Clio, 1996/4, nº spécial « Le temps des jeunes filles ", 
[en ligne], URL :

http://clio.revues.org/ document432.html, consulté le 26 avril 2007.

(15) Hugo the

Schepper, «The Individual on Trial in the Sixteenth-

Century Netherlands between Tradition and

Modernity ", in Charles

H. Parker, Jerry H.

Bentley, [dir.], Between the Middle Ages and

Modernity : Individual and Community in the Early

Modern World. Essays

dedicated to James Tracy,

Lanham, Rowman

\& Liddlefield, 2007,

p. 187-209.

(16) Herman Pleij,

Restyling Wisdom,

"Remodelling the Nobi-

lity, and Caricaturing

the Peasant : Urban

Literature in the Late

Medieval Low Countries ",

in Journal of Interdis-

ciplinary History, 32-4,

2002, p. 689-704 ; Heinz

Schilling, Lars Behrisch,

[dir.], Institutions,
Instruments and Agents of

Social Control and

Discipline in Early Modern

Europe, Francfort am

Main, Vittorio Kloster-

mann, 1999 ; Herman

Roodenburg, Pieter

Spierenburg, [dir.], Social

Control in Europe, Volume

I, I500-1800, Columbus,

Ohio UP, 2004.

(17) Bernard Schnapper, " La répression du vagabondage et sa signification historique du XIVème au XVIIIème siècle ", Revue d'histoire du droit français et étranger, 63, 1985, p. 143-157.

(18) Joos de Damhoudere, La practique et enchiridion des causes criminelles, Louvain, 1555.
La criminalisation de l'homicide par les pouvoirs centraux s'inscrit dans ce processus. Le criminaliste Joos de Damhoudere, homme du Prince, mais citoyen brugeois, définit dorénavant l'homicide comme « le deuxième crime qui naquit sur la terre, après la prévarication et la désobéissance d'Adam et Ève [...] ". ${ }^{(18)}$ Les lettres de rémission qui concernent beaucoup d'hommes jeunes renforcent ce mouvement en tendant à détacher l'action de l'individu de son contexte collectif et en imposant la définition du pardonné comme sujet du prince et non plus comme membre du lignage. On peut penser que cette pression a accentué le rôle des fameuses " abbayes de jeunesse » et confréries par classes d'âge. Les réseaux d'âges se superposent aux réseaux lignagers et familiaux. La jeunesse devient alors un temps spécifique, à encadrer comme tel. La violence des jeunes est mise au service des adultes dans une optique de contrôle des mœurs déviantes. On peut considérer l'autonomisation des confréries de jeunesse comme une manifestation de la transition entre une société de clans, fondée sur le lignage et la reproduction, et une société d'individus, plus mobile. La catégorie jeunesse deviendrait un " temps social " propre, où les jeunes s'exprimeraient hors des relations de lignage.

Ce mouvement se combine et s'enracine dans le « temps des réformes ». La période 1450-1650 y apparaît non seulement comme la fin du mythe de la Chrétienté médiévale, la confessionnalisation des partis ou encore la vaste recomposition socioéconomique du capitalisme émergeant, mais également comme le temps d'une entreprise massive de transformation des comportements et des mentalités urbaines. C'est ce qu'exprime, le 29 juin 1572, le jésuite Jean Balé dans une missive à son supérieur général : "À la suite des maux de toute 
sorte, des guerres, des hérésies, qui chaque jour nous menacent, la jeunesse de notre temps devient sauvage, indomptée, intraitable et belliqueuse. " (19) Cette phrase décrit l'atmosphère de guerre civile et de démoralisation qui règne dans une des régions les plus prospères d'Europe en cette fin de XVIème siècle, les Pays-Bas. Catholiques et Réformés découvrent les uns et les autres avec angoisse que leur société est menacée de l'intérieur par divers maux (violence, " sectes sataniques ", dissolution des mœurs). Les grands " réformateurs " s'attaqueront de front à cette jeunesse indisciplinée, autour du diptyque violence et prostitution. Nombre de grands réformateurs, parmi lesquels Martin Luther, Jean Calvin et Iñigo de Loyola, furent des concepteurs ou promoteurs d'ordonnances publiques contre la prostitution, s'inscrivant dans le courant des « Policey Ordnungen " dans toutes les villes d'Europe. ${ }^{(20)}$

Dans le monde urbain, les métiers concentraient à la fois les risques et les moyens de les endiguer. Chez les catholiques, les jésuites élaboreront ainsi un modèle particulièrement développé de transformation des confréries médiévales en instrument de création de ce que Robert Muchembled a appelé « un homme nouveau, maitre de lui-même dans ses attitudes corporelles, sa vie familiale ou son intimité domestique, [qui] se façonne dans cette culture d'intense culpabilisation personnelle et collective ". ${ }^{(21)}$

Dans un contexte où les sociabilités urbaines sont soumises à la pression d'un pouvoir central en développement (l'État), la scolarisation des élites nobles et bourgeoises devient l'instrument d'une transformation des valeurs de la jeunesse. Dans les collèges, patriotisme monarchique, piété intérieure et politesse deviennent les trois vertus privilégiées. Ainsi, l'élève du collège jésuite de 1620 est enserré dans un système disciplinaire strict qui contraste avec l'atmosphère libérale du XVIème siècle. Les absences sont rigoureusement contrôlées. Il est défendu d'entrer au collège avec des armes, poignards, épées, qui sont déposées à l'entrée dans la " chambre des armes ». Défense également de dégrader les murs, les portes, les bancs, le matériel scolaire, d'y tracer ou d'y graver des inscriptions.

Hors du collège, le règlement prévoit une discipline aussi sévère. Il n'est pas permis de glisser sur la glace en novembre, de se baigner en mai. Aux jours de congé, il est déconseillé de jouer dans les rues, mais conseillé de se réunir en des endroits déterminés pour jouer en parlant latin. Pas question
(19) Cité dans Albert Poncelet, Histoire de la Compagnie de Jésus dans les anciens Pays-Bas. Établissement de la Compagnie de Jésus en Belgique et ses développements jusquià la fin du règne d'Albert et d'Isabelle, t. 2., Bruxelles, Hayez, 1927, ch. 12, p. 321-345.

(20) Les ordonnances de police caractérisent un courant de législation qui se développe dans de nombreuses villes d'Europe fin du XVème siècle, définissant un domaine de la régulation sociale distinct de la justice : la police. On y retrouve des prescriptions sur la vie économique, le commerce, la santé et l'ordre public. Karl Härter, [dir.], Policey und frühneuzeitliche Gesellschaft, Frankfurt am Main, Vittorio Klostermann, 2000 ; Paolo Napoli, Naissance de la police moderne. Pouvoir, normes, sociétés, Paris, La découverte, 2003. 
(21) Louis Châtellier, L'Europe des dévots, Paris, Flammarion, 1987.

(22) Natalie Zemon Davis, Les cultures $d u$ peuple, rituels, savoirs et résistances au XVIème siècle, Paris, Aubier, 1979.

(23) Louis Châtellier, La religion des pauvres : les missions rurales en Europe et la formation du catholicisme moderne, XVIème-XIXème siècle, Paris, Aubier, 1993. d'assister à des représentations ni à des jeux publics, ni de tenir un rôle dans un théâtre de la ville, sauf autorisation spéciale. Les jeux de cartes et jeux d'argent sont interdits. Pas question non plus d'assister aux supplices des condamnés à mort, sauf peut-être pour crime d'hérésie, admet cependant le règlement des collèges.

Ce modèle d'intériorisation des affects s'inscrit bien dans le processus suggéré par Norbert Élias " de civilisation des mœurs ». Il invite à dépasser la querelle entre partisans d'une lecture répressive de la civilisation, processus imposé par les élites aux populations rétives, et ceux d'une lecture consensuelle de la civilisation, processus lié au développement de la culture urbaine partagée. Les mutations de la société occidentale aux XVème-XVIIème siècles me semblent à la fois combiner une pression des autorités (disciplinarisation, réveil éthique) et une évolution culturelle interne (individualisation, autocontrôle). Les comportements des jeunes sont à la fois l'enjeu et le vecteur de cette mutation. Progressivement soumis par la scolarisation à une disqualification de la violence, les jeunes sont l'objet d'un encadrement plus strict par les adultes. Chiens de garde de l'ordre moral à travers les abbayes de jeunesse, les charivaris et les rituels de festivités, ils contribuent à répandre ce modèle de la disqualification des écarts sexuels ou moraux, tout en obtenant de faire usage d'une violence ritualisée. ${ }^{(22)}$ Dans les sociétés urbaines, le processus de civilisation des mœurs s'appuie sur un processus de recomposition des rôles entre jeunes et adultes. Dans leur volonté de dominer la hiérarchie sociale, les élites bourgeoises s'appuient également sur une restructuration des groupes d'âge. Le vaste mouvement de christianisation des campagnes entrepris par les missionnaires catholiques et réformés aux XVIIème et XVIIIème siècles répand ce modèle dans les campagnes. De la Bretagne au Luxembourg, les « missions rurales " diffusent ce modèle de " civilisation des mœurs ".

Bien entendu, les jeunes font de la résistance, notamment les confréries et abbayes de jeunesse, figures de l'insubordination et de la révolte. Mais parmi les figures de la rébellion, les conflits entre jeunes et adultes se manifestent clairement à la fin de l'Ancien Régime. Les archives judiciaires recensent nombre de charivaris et de pratiques populaires qui aboutissent à un conflit frontal avec les autorités locales, surtout dans les villages. Les uns et les autres s'appuient alors sur un acteur nouveau, la justice princière, pour obtenir le 
maintien de leurs traditions séculaires ou la « fin de ces excès scandaleux ». Les

(24) Étienne Cléda,

Justice et ordre moral. Discours des procureurs généraux du Conseil provincial de Namur sur les comportements sexuels au XVIIème siècle,

Louvain-La-Neuve, UCL, 1999, mémoire de licence en histoire, inédit.

(25) Xavier Rousseaux, "L'incrimination du vagabondage en Brabant (XIVème-XVIIIème siècles). Langages du droit et réalités de la pratique ", in Langage et droit à travers l'histoire. Réalités et fictions, Louvain-Paris,

Nauwelaers, 1989, p. 147183 ; Pieter Spierenburg, [dir.], Men and Violence. Gender, Honor and Rituals in Modern Europe and America, Columbus, Ohio UP, 1998. 
(26) Voir les travaux de Joanna Bourke, A Intimate History of Killings. Face to face Killing in XXth Century Warfare, New York, Basic Books, 1999.

(27) Georges Mossé, De la Grande Guerre au totalitarisme. La brutalisation des sociétés européennes, Paris, HachetteLittératures, 1999.

(28) Dans la plupart des pays d'Europe, ce mouvement démarre dans les années 1880 sur le plan législatif et institutionnel, mais n'est réellement appliqué à l'ensemble des classes d'âges que dans les premières décennies du XXème siècle. axe est celui de conjonctures particulières : celle d'un demi-siècle où la canalisation de la violence de la jeunesse devient une question d'État.

De 1860 à 1950, les conflits croissants entre États-nations en Europe semblent mobiliser la jeunesse dans un double processus : une pacification à l'intérieur des frontières de la Nation et une «brutalisation » contrôlée, à travers le service militaire, vers l'extérieur. On assiste en même temps à la " politisation " des violences collectives au plan local et à la « militarisation » des violences juvéniles au plan national. On peut cependant faire l'hypothèse que ces formes d'encadrement de la violence juvénile demeurent instables, lorsque l'État est affaibli par des dissensions internes ou des conflits extérieurs.

D'une part, la pacification se marque dans la diminution d'intensité et de nombre des formes collectives de violence juvénile. François Ploux l'observe pour le Sud-Ouest, Aurélien Lignereux dans les rébellions à gendarmes. Le premier évoque le lien avec " la mise en place, notamment sous la IIIème République, d'un réseau d'associations communales spécialisées dans le loisir ».

D'autre part, le contrôle de la force physique se manifeste dans les rivalités internationales et culmine dans la figure du service militaire obligatoire pour les jeunes hommes. Or, apprendre à donner la mort légitimement, alors que le processus de socialisation vise à susciter l'horreur de la violence, entrầne un paradoxe insoutenable pour de nombreux jeunes enrôlés dans les guerres du XXème siècle. ${ }^{(26)}$

Au sortir de la " der des der ", les adultes, traumatisés par les boucheries de la Grande Guerre, développent une hantise comparable à celles des réformateurs du XVIème siècle face à l'indiscipline des jeunes, doublée de la peur du déclin démographique. ${ }^{(27)}$

L'entre-deux-guerres est la période de généralisation du " grand encadrement " des jeunes. ${ }^{(28)}$ Par l'école obligatoire tout d'abord, qui étend son filet dans le temps : du lundi au samedi et de 7 à 14 ans. Par les mouvements de jeunesse, qui organisent le temps non scolaire : soirées, dimanches et "vacances ». Par la surveillance croissante de la natalité et l'attention portée à la petite enfance. Quel que soit le projet idéologique, les méthodes, les modèles, les formes se ressemblent. À la différence de l'entreprise réformatrice du XVIème siècle, durant l'entre-deux-guerres, il ne s'agit pas de transformer une sociabilité collective médiévale " clanique » en une autre « individualisée ", modernisée et urbanisée. 
Au contraire, face aux progrès de l'individualisme, nombre d'acteurs sociaux plaident pour le développement d'une sociabilité collective pour faire pièce à l'individualisme égocentré, nouvelle figure de la destruction de la société. Syndicats, églises, partis politiques, associations culturelles, entrepreneurs moraux et minorités culturelles investissent dans les mécanismes d'intégration de la jeunesse dans le projet collectif. La figure limite en est le mouvement de jeunesse unique, tel qu’il sera réalisé dans les régimes totalitaires à l'Est comme à l'Ouest.

La sortie de la seconde guerre correspond à une profonde crise sur le rôle des jeunes dans la société. Un indice se trouve dans leur surcriminalisation durant les événements de la guerre. Non seulement les jeunes meurent davantage que les plus âgés sur les champs de bataille, mais ils subissent davantage les répressions. Car, pour la plupart des belligérants, l'expérience des violences guerrières se double d'une expérience nouvelle (comparable à celle de la première guerre pour les Belges et les habitants du Nord-Pas-de-Calais), celle de l'occupation. Ivan Jablonka remarque combien, pendant les deux guerres, « la surveillance des adultes sur les enfants se relâche ». Face aux jeunes adultes mobilisés par l'effort de guerre, les adolescents sont laissés sans repères. En outre, dans les régions occupées, le mélange « culturel » affaiblit également l'autorité parentale, soit en raison de l'absence du père (prisonnier, déporté, résistant), soit par la "puissance » des militaires occupants qui décrédibilise l'autorité civile du père jusqu'au cour des familles. ${ }^{(29)}$ Il en résulte une insolence plus grande prêtée aux jeunes garçons et filles. Marché noir et vols sont attribués aux bandes de jeunes, fréquentations sexuelles aux filles. Ces nouvelles «menaces" justifient une préoccupation croissante des familles comme des autorités quant à la protection des jeunes menacés par la contamination idéologique ou physique, et suscite la crainte d'une insubordination générale de la jeunesse, que certains verront éclater en mai 1968.

Le débat sur le sort à réserver aux mineurs collaborateurs déchira les résistants partout en Europe. ${ }^{(30)}$ Jérôme de Brouwer nous rappelle que la Belgique, chantre du libéralisme pénal, voulut limiter l'usage de la peine de mort et en fit, au XIXème siècle, un interdit pour les mineurs de moins de 21 ans. Or, en 1945, plusieurs mineurs figurent parmi les condamnés à mort de la répression de la collaboration menée par les autorités belges. Un avocat, Pierre Vermeylen, s'indigne dans la presse de l'oubli du droit que provoque la soif de

(29) C'est également la lecture que fait Fabrice Virgili des tontes de la sortie de deuxième guerre, qui symbolise la revanche sur une masculinité humiliée par la défaite de 1940, laquelle se double d'un problème de génération : les jeunes de 1945 tentant de compenser la faillite de leurs aînés. Fabrice Virgili, La France "virile ». Des femmes tondues à la Libération, Paris, Payot, 2000.

(30) En Maine-et-Loire, sur le plan quantitatif, les jeunes collaborateurs ne semblent pas davantage poursuivis que les autres. Cela dit, un examen qualitatif indique qu'ils sont plus jusqu'au boutistes, et donc plus sévèrement réprimés, pour des motifs proprement politiques. Marc Bergère, Une société en épuration : épuration vécue et perçue en Maine-etLoire, de la Libération au début des années I950, Rennes, Presses univer- 
sitaires de Rennes, 2004, p. 140-141.

(31) Ce néologisme est l'acclimatation de "kidult ", formule américaine des années 1980 . Plus largement, s'opère en ce moment tout un redécoupage des âges de la vie, avec des catégories beaucoup plus fines : petite enfance, enfance, " adonaissants » - voir François de Singly, Les adonaissants. Les connaissezvous vraiment?, Paris, Armand Colin, 2006 -, " adolescents ", " adulescents »... Le schème enfant/adulte en devient de plus en plus trouble. Voir aussi Alessandro Cavalli, Olivier Galland, [dir.], L'allongement de la jeunesse, Arles, Actes Sud, 1993. vengeance. Il réagit à l'annonce de la condamnation à mort d'un mineur dans un procès de collaborateurs, procès mené devant un tribunal présidé par un juge pour enfants, résistant... Cela n'empêchera pas certains d'entre eux de passer devant le peloton d'exécution.

Conscients des dérives idéologiques particulières du conflit, les autorités publiques tentent partout de contrôler les faits et gestes de la jeunesse. L'extension de l'obligation scolaire vers le haut (jusqu'à 16, voire 18 ans) et vers le bas (maternelle), le maintien d'une conscription modernisée, les tentatives de censurer les publications destinées à la jeunesse, s'accompagnent d'une véritable prise en charge de l'enfance irrégulière par l'État social. La reconstruction et le plein-emploi huilent le système, permettant aux jeunesses populaires et bourgeoises de s'enrichir et de bénéficier à plein de la consommation de masse. Ce modèle se retrouve en creux dans les aspirations et la mobilité des filles décrites par Anne Thomazeau.

Depuis la fin des années 1970, la crise économique durable en Europe a grippé le mécanisme de l'ascenseur social pour les jeunes. Les jeunesses se retrouvent progressivement désencadrées par les grandes institutions socialisatrices, autonomisées comme groupe économique par la société de consommation, prolongées dans un système de formation bureaucratique jusqu'au milieu de la vingtaine d'années.

Ressurgit alors la vieille peur du monde adulte, accentuée par les effets présumés du vieillissement des populations, de voir une jeunesse violente s'attaquer à des adultes fragilisés. Un symbole en est le débat sur les quartiers résidentiels, réservés aux retraités, interdits aux jeunes familles, aux enfants et aux adolescents, qui se multiplient dans les pays anglo-saxons. Adultes vieillissants s'opposent à des jeunes de plus en plus... " adulescents ». ${ }^{.31)}$

Cette vision cavalière des rapports en dents de scie entre adultes et jeunes est trop générale pour expliquer les situations précises. Elle indique cependant que « la violence des jeunes » est essentiellement la violence prêtée aux jeunes par les adultes. Phénomène intéressant, la bourgeoisie apparaît bien l'acteur déterminant dans l'intérêt pour le contrôle de la jeunesse. La noblesse juvénile, éduquée dans la culture de la violence, sera la plus lente à désarmer et à subir l'interdit progressif du duel. Quant aux classes populaires, au village, en ville et à l'usine, elles subissent des encadrements distincts. Progressivement s'élabore une pola- 
risation entre classes bourgeoises et classes populaires. Les premiers investissent les réseaux privés comme l'État pour mettre à l'agenda politique la surveillance des jeunes populaires. Après la seconde guerre, l'exode rural sonne le déclin du modèle communautaire, tandis que la crise post-industrielle des années 1970 disloque l'encadrement de la classe ouvrière.

En deçà d'une vision macrohistorique, les études de cas présentées dans ce numéro soulignent également le flou des concepts de "violence " et de " jeunesse ». Dans le domaine juridique : la qualification de violence est presque indissolublement liée à celle $\mathrm{d}^{\prime}$ " indiscipline ». On reproche rarement à un vieux pervers d'être indiscipliné. Anne Thomazeau montre que la violence physique est peu présente dans les maisons de correction pour filles, en revanche les figures de la rébellion l'évoquent en creux. Ivan Jablonka signale combien la cruauté sur les animaux est perçue par les adultes comme un signe de perversité du jeune. Les violences des jeunes placés à l'Assistance ou à la Protection judiciaire de la jeunesse au XXème siècle se transforment en désobéissances. Le poids de la parole devient plus important que les gestes. Ceci indique également combien les jeunes savent « jusqu'où aller trop loin "...

Quant à la notion de jeunesse, elle est également variable. La construction historique de la délinquance juvénile est un phénomène européen, voire occidental. Entre 1790 et 1840, la spécificité de la jeunesse fut d'abord élaborée dans le débat sur la majorité (15, 16, 18, 21 ans) et par l'angle de l'enfermement avec la création de maisons spécialisées pour mineurs. ${ }^{(32)}$ Le gavroche est le symbole des jeunes vagabonds ruraux arrivés en ville. Dans la seconde moitié du siècle, le jeune ouvrier remplace le vagabond issu des campagnes dans les fantasmes de la classe bourgeoise. Oliver Twist remplace Gavroche, avec son passé de maison de correction. Les «Scuttlers» de Manchester, les "Hooligans» londoniens, les "Apaches" parisiens traduisent l'ambiguïté du mythe. Ces figures décrivent des groupes et des pratiques de jeunes en bande, mais évoque des représentations stratifiées d'une sociabilité déviante des entrepôts, des stades ou des fortif' par des bourgeois qui ne s'y déplacent que rarement.

Les différentes contributions apportent enfin une précision croissante de la chronologie des violences juvéniles au moins pour les deux derniers siècles. Le mouvement décroissant touche aussi bien les violences attribuées aux jeunes que les violences des adultes. Mais les formes sont différentes. Le glis-
(32) Peter King, « The

Rise of Juvenile

Delinquency in England 1780-1840, Changing Patterns of Perception and Prosecution ", Past and Present, 160, 1998, p. 116-166; Pamela Cox, Heather Shore, [dir.], Becoming Delinquent: British and European Youth, I650-I95o, Alderhot, Ashgate, 2002. 
(33) Antoon Vrints le

constate dans une très fine étude sur la violence à

Anvers des années 1910 à

1950, basée sur les

procès-verbaux de police.

Het theater van de straat.

Publiek geweld, respecta-

biliteit en sociabiliteit in

Antwerpen (ca I9I0-I950)

[Le théâtre de la rue.

Violence publique, respec-

tabilité et sociabilité à

Anvers, env. 1910-1950],

Gand, université de Gand,

2006 (thèse de doctorat

en histoire, inédite).

(34) Ginette Kurgan-

Van Hentenrijk, [dir.],

Un pays si tranquille? La

violence en Belgique au

XIXème siècle, Bruxelles,

éditions de l'ULB, 1999. sement de la répression de l'homicide vers les coups et blessures ne met pas en cause le fait que la violence physique dans l'espace public demeure une attitude de jeunes mâles. ${ }^{(33)}$ En matière de violence verbale, la situation est plus contrastée. Quoi qu'il en soit, sur la longue durée, l'utilisation de la force physique comme moyen d'expression, de communication dans un conflit diminue avec les processus d'encadrement décrits plus haut. La violence interne ou externe des jeunes dans les campagnes du Sud-Ouest, la rébellion face aux gendarmes à la même époque évoquent une transformation dans la seconde moitié du XIXème siècle en France. La violence physique grave, qui fait couler le sang, décroît régulièrement. Pourtant, les poursuites et la répression de la violence ne font que croître. De nouvelles figures se dessinent : la violence verbale et les rébellions à agent mobilisent les polices urbaines dans la seconde moitié du XIXème siècle, en destination particulière des jeunes. ${ }^{(34)}$

En résumé, comme le suggèrent les textes de ce dossier, les définitions, les formes et les perceptions de la violence comme de la jeunesse ont évolué de manière non linéaire en Occident. Bien des recherches sont encore nécessaires pour en discerner les liaisons dangereuses. 